\title{
False Positive FDG-PET Scan Caused by Uterine Leiomyoma
}

Gergely Varga ${ }^{1}$, Hajna Galgoczy ${ }^{2}$, Peter Szabo ${ }^{2}$, Ferenc Toth $^{3}$, Csaba Diczhazi ${ }^{4}$ and Peter Farkas ${ }^{1}$

${ }^{1} 3$ rd Department of Internal Medicine, Semmelweis University, Budapest, Hungary

${ }^{2}$ PET/CT Unit, ScanoMed Ltd., Budapest, Hungary

${ }^{3}$ Department of Gynaecology, Semmelweis University, Budapest, Hungary

${ }^{4} 1$ st Department of Pathology and Experimental Cancer Research, Budapest, Hungary

*Corresponding author: Gergely Varga, 3rd Department of Internal Medicine, Semmelweis University, 4 Kutvolgyi Street, H-1125, Budapest, Hungary, Tel: 36208258799; E-mail: vargager@gmail.com

Received date: Aug 07, 2015; Accepted date: Oct 28, 2015; Published date: Oct 30, 2015

Copyright: (c) 2015 Varga G, et al. This is an open-access article distributed under the terms of the Creative Commons Attribution License, which permits unrestricted use, distribution, and reproduction in any medium, provided the original author and source are credited.

\begin{abstract}
Positron emission tomography with 2-deoxy-2-[fluorine-18]fluoro- D-glucose (18F-FDG-PET) has an important role in the management of lymphoma, but it is important to keep in mind that not only lymphoma but also a number of other pathologic and non-pathologic processes can cause increased tissue FDG uptake. Our case is a rare example when a uterine fibroid caused differential diagnostic problem.
\end{abstract}

Keywords: Lymphoma; Diffuse large B-cell lymphoma; Positron emission tomography; Uterine fibroid; Uterine leiomyoma

\section{Introduction}

In the last 10 years positron emission tomography with 2-deoxy-2[fluorine-18] fluoro-D-glucose (18F-FDG-PET) has emerged as a powerful imaging tool for the detection of various cancers. In high grade lymphoma it helps accurate staging as well as restaging post chemotherapy as it is able to identify nodal and extranodal sites with greater senstivity than computer tomography (CT) alone [1]. Persistent post-chemotherapy masses are common differential diagnostic problems in lymphoma when the level of FDG avidity often provides a clue to clinical management.

\section{Case Report}

A 45-year-old woman presented with marked B symptoms, splenomegaly and severe pancytopenia. Her bone marrow trephine biopsy confirmed heavy infiltration of diffuse large B-cell lymphoma; the staging CT scan showed no nodal involvement (stage 4, IPI 2). She went on to have rituximab-cyclophosphamide-doxorubicin-vincristinprednisolon (R-CHOP) chemotherapy resulting in the improvement of her blood counts, the disappearance of her B symptoms and the resolution of the splenomegaly.

At the end of her planned 6 cycles of treatment the bone marrow trephine confirmed remission, and her FDG-PET showed no abnormalities in the regions previously involved, however revealed a highly active $(26 \times 27 \times 30 \mathrm{~mm}$, weight normalized SUVmax: $9.0 \mathrm{~g} / \mathrm{ml})$ nodule attached to the uterus (Figure 1, MIP image). Given that the in addition to persistent lymphoma the differential included leiomyosarcoma hysterectomy was performed. Histopathologically the tumor was highly vascularized uterine leiomyoma (Figure 2, HE, 250X). No further treatment was utilized, she is alive and well 2 years post chemotherapy.

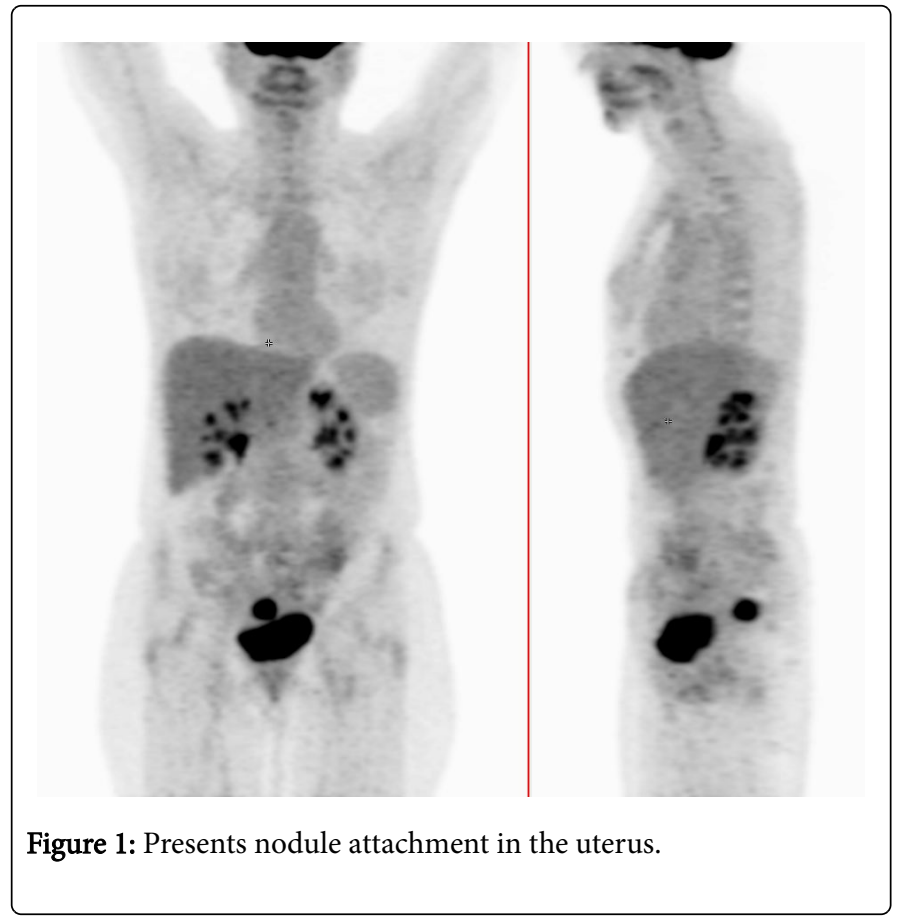

\section{Discussion}

FDG-PET have radically changed the management of lymphoma. Growing evidence supports its incorporation into clinical decision algorithms, negative interim and end of treatment PET being strong predictors of good outcome in most lymphoma subtypes. As a result of its widespread use, false PET positivity became a common challenge. Examples include infection, post chemo- or radiotherapy inflammation and not uncommonly secondary tumors both benign an malignant [2-4].

Uterine fibroid, a benign tumor composed mainly of smooth muscle cells, is typically negative on PET. PET can actually be used to 
Citation: $\quad$ Varga G, Galgoczy H, Szabo P, Toth F, Diczhazi C, et al. (2015) False Positive FDG-PET Scan Caused by Uterine Leiomyoma. J Cytol

Page 2 of 2

differentiate leiomyomas from leiomyosarcomas [5]. Although this is a rare exception from the normal behavior of uterine fibroids our experience demonstrates that the differential for pelvic FDG avid lesions should include uterine fibroids.

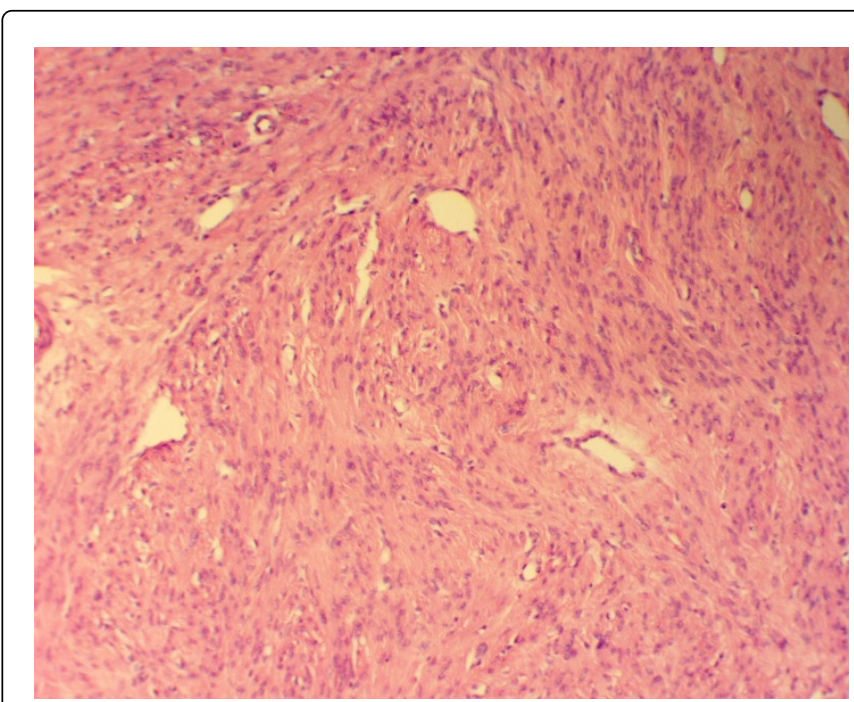

Figure 2: Histopathological view of the vascularized leiyomyoma.

\section{Authors' contribution}

Gergely Varga and Peter Farkas treated the patient and wrote the text; Hajna Galgoczy and Peter Szabo reported the PET scans; Ferenc Toth did the US follow up and performed the surgery, Csaba Diczhazi did the histopathology. All authored have read and authorized the text.

\section{Conflict of interest}

Authors declare that there are no competing financial interests in relation to the work described.

\section{References}

1. Mylam KJ, Nielsen AL, Pedersen LM, Hutchings M (2014) Fluorine-18fluorodeoxyglucose Positron Emission Tomography in Diffuse Large Bcell Lymphoma. PET Clin 9: 443-455, vi.

2. Culverwell AD, Scarsbrook AF, Chowdhury FU (2011) False-positive uptake on 2-[1 $8 \mathrm{~F}]$-fluoro-2-deoxy-D-glucose (FDG) positron-emission tomography/computed tomography (PET/CT) in oncological imaging. Clin Radiol 66: 366-82.

3. Wang X, Koch S (2009) Positron emission tomography/computed tomography potential pitfalls and artifacts. Curr Probl Diagn Radiol 38: 156-169.

4. Cook GJ, Wegner EA, Fogelman I (2004) Pitfalls and artifacts in 18FDG PET and PET/CT oncologic imaging. Semin Nucl Med 34: 122-133.

5. Kitajima K, Murakami K, Kaji Y, Sugimura K (2010) Spectrum of FDG PET/CT findings of uterine tumors. AJR Am J Roentgenol 195: 737-743. 